\title{
Towards Attosecond Pulses with High Harmonics
}

\author{
P. Agostini ${ }^{a}$, P.M. Paul ${ }^{a}$, P. Breger ${ }^{a}$, E.S. Toma ${ }^{b}$, \\ H.G. Muller ${ }^{b}$, G. Mullot ${ }^{c}$, F. Augéc and Ph. Balcou \\ ${ }^{a}$ Commissariat á l'Energie Atomique, Centre d'Etudes de Saclay \\ 91191 Gif sur Yvette, France \\ ${ }^{b}$ FOM AMOLF, Kruislaan 407, 1098 SJ Amsterdam, The Netherlands \\ ${ }^{c}$ Laboratoire d'Optique Appliquée, ENSTA, Ecole Polytechnique \\ CNRS UMR 763991761 Palaiseau Cedex, France
}

\begin{abstract}
The high harmonics produced by focusing an intense femtosecond laser in a gas are theoretically shown to be locked in phase. The physics of this locking is discussed and a new method based on quantum interference in two-photon, two-color ionization allowing to retrieve the relative phase of harmonic pairs is described. The main result is that the 5 harmonics of orders 11-19 produced in argon generate a train of subfemtosecond pulses with a period of $1.35 \mathrm{fs}$ and a duration of 250 attoseconds.
\end{abstract}

PACS numbers: 42.65.Re, 42.65.Ky, 32.80.Rm, 33.60.Cv

\section{Introduction}

The race to shorter light pulses has started immediately after the advent of the laser in 1960. With the invention of the $Q$-switching by Bob Hellwarth, in 1964, pulses dropped from 100 microseconds to 100 nanoseconds. The introduction of the mode-locking by DeMaria, shrank the pulse lengths another five orders of magnitude to $1 \mathrm{ps}$. This tremendous reduction of factor $10^{8}$ occurred in just a few years. The pace then considerably slowed down and it took about 10 years to Shank and Ippen to cross the picosecond line through intra-cavity compensation of the group-velocity dispersion in a cw mode-locked oscillator. The next steps were even more painstakingly slow. The invention of the chirped pulse amplification method by G. Mourou launched the era of ultra-short ultra-intense all-solid-state lasers but did little to the pulse shortening itself. The discovery of the Kerr lens mode-locking (known for a while as the magic mode-locking, until the mechanism was elucidated) 
in a Ti:sapphire oscillator by Sibbet in 1991 triggered a new descent although a very slow one: in spite of the introduction of the chirped mirrors and techniques to produce large bandwidths through self-phase modulation the shortest pulse for a Ti:sapphire laser as yet is still about $5 \mathrm{fs}$ and the rate of decrease, on the scale of Fig. 1, is close to zero. There are several alternatives to the Ti:sapphire laser itself though, carrying the promise to break the femtosecond barrier: high-harmonic

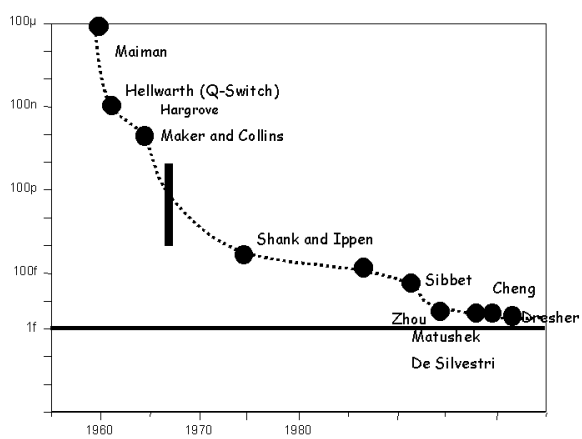

Fig. 1. Steps of the laser short pulses history. The 1 fs line seems hard to cross. References to the original articles may be found in the excellent review [1].

generated (HHG) by an ultrashort ( $7 \mathrm{fs}$ ) $800 \mathrm{~nm}$ pulse was already shown to be a good candidate for crossing the line [2]. The bandwidth stretching through refractive index modulation in a molecular gas induced by driving a Raman transition slightly off-resonance [3] is surely most promising too and offers a number of very attractive features: high conversion efficiency, wide bandwidth, direct control of the phases of the successive Stokes components thus allowing the production and shaping of subfemtosecond pulses. The present work is dealing with still another possible route to such pulses which utilizes the bandwidth of the high harmonics spectrum and the (surprising) theoretically-predicted property of a constant phase relationship between the spectral components of the plateau. The temporal beating of equally spaced quasi-monochromatic waves would then generate a periodic train of pulses, each of which with a duration of a few optical cycles only. However, a nonlinear, instead of linear, spectral phase density (not to mention a random one) would be sufficient to result only in a very weak modulation of the field amplitude.

Although high harmonics have been studied for more than fifteen years and are commonly utilized as compact source of XUV radiation [4], the question of their relative phases has been addressed only in theory [5] because of the experimental difficulty in measuring the beating in the time-domain. There is no known method to resolve such sharp temporal substructures, if they exist, except autocorrelation. Unfortunately autocorrelation requires a nonlinear signal which harmonics have, as yet, proved unable to provide except for low orders [6]. A special property 
of two-photon, multicolor ionization known for sometime (Véniard et al. [7]) is able nevertheless to provide the desired information in the frequency domain. In a recent work [8] we have implemented this method and indeed observed a linear spectral phase density in a group of five harmonics generated in argon, as predicted in [5] with the exciting consequence that pulses as short as 250 as are produced by such a superposition.

In the present paper we shall first review the theoretical reasons why harmonics phases may be "locked", then summarize the Véniard et al. method and how we have applied it to the harmonic phase determination. We shall briefly discuss the results, their extrapolation to even shorter pulses and finally the possibility to select a single subfemtosecond harmonic pulse.

\section{Why are high harmonics in phase?}

The generation of high order harmonics is very different from the familiar situation of nonlinear optics: in this latter case the electron remains strongly bound and emission is that of a slightly anharmonic oscillator. In HHG, on the contrary, an electron wave packet appears in the continuum at some distance of the nucleus in a fraction of the pump laser optical cycle. The fate of this wave packet depends on the phase of the pump field at which it has been freed: liberated before a maximum of the field, it will never return (ionization). Wave packets born in the continuum after the maximum of the field will, on the contrary, be driven back more or less rapidly by the field to the nucleus and recombine to the ground state or rescatter from it (Fig. 2). This is the essence of a very popular classical model of HHG [9] and its quantum extension first worked out by Lewenstein et al. [10].

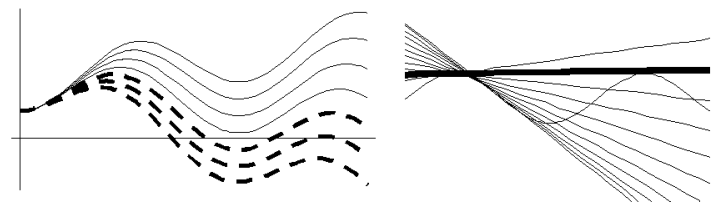

Fig. 2. Classical electron trajectories in the lab frame (left) in the Kramers-Henneberger oscillating frame (right). Trajectories returning to the nucleus are shown in dashed lines (left) or below the thick line (right).

The classical electron trajectories in the electromagnetic field are the basis of the physics of HHG. Such trajectories can be visualized in the lab frame or, more clearly in the frame oscillating at the optical frequency in which the electron is either at rest or travels in a straight lines with an initial velocity such that the line is tangent to the sine curve which, in this frame, shows the trajectory of the nucleus. This representation in this so-called Kramers-Henneberger (or KH) frame was first 
introduced by Muller [11]. One of the distinguishing features of the Corkum model is that it predicts immediately the value of the highest harmonic order (cutoff) which can be produced at a given pump intensity from energy conservation as the sum of the maximum kinetic energy the electron can have at the moment of the recollision with the nucleus $\left(3.17 U_{\mathrm{p}}\right.$, where $U_{\mathrm{p}}$ is the ponderomotive energy) and the atom ionization potential $I_{\mathrm{p}}$. Besides, the model has the interesting and not trivial consequence that the atomic dipole is complex and has a phase given by the quasi-classical action between the instant the electron leaves the nucleus and that of its return and recombination [10, 12].

For each harmonic (corresponding to a given kinetic energy of the re-colliding electron) there are two trajectories (shown in the KH frame in Fig. 3).

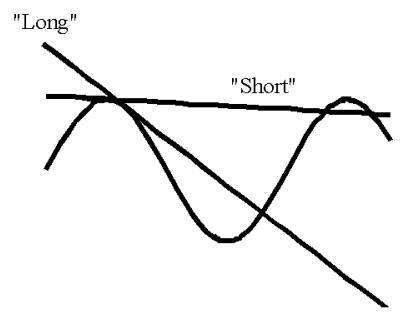

Fig. 3. Long and short trajectories (straight lines) with the same kinetic energy upon return to the nucleus (sine curve).

Antoine et al. [5] have calculated those phases for consecutive harmonics and judged that a plot of the phase differences versus the harmonic order looked pretty much random. Proceeding to the calculation of the superposition of harmonics with such phases (and amplitudes derived from the same model [10]) they discover that "... surprisingly, the train of pulses is not as irregular as one could have expected on the basis of the phase variation". We would like to remark that the calculated phase differences may not be as irregular as it appears at first glance and confirm that the underlying regularity is indeed a consequence of the classical motion of the electron.

The remark is sustained by the graph of Fig. 4 which reveals a regularity if one considers two groups: for each group the phase difference is approximately a linear function of the order. If $a$ is the slope of such a function, the modulus square of the sum of $N$ harmonics is the function $\csc ^{2}(\omega t+a) \sin ^{2} N(\omega t+a)$ which has two temporal peaks per fundamental cycle $2 \pi / \omega$. The other group with a slope $b$ will also produce two peaks per optical cycle phase-shifted with respect to the first ones by $(b-a)$. Therefore, a phase distribution as the one in Fig. 3 will in general produce 4 narrow peaks per optical cycle as indeed found in [5]. One can verify that the phases derived from the purely classical trajectories are distributed along a curve similar to the one calculated from the Lewenstein model in showing the quantity $-U_{\mathrm{p}} \tau$ as a function of the kinetic return energy (Fig. 5). 


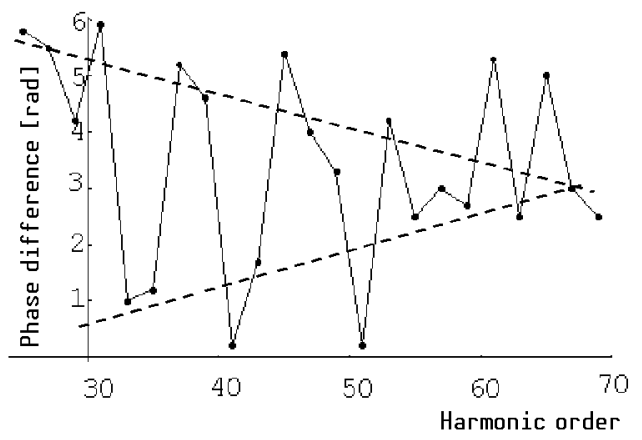

Fig. 4. Phases difference vs. harmonic order. The dots are the values taken from [5]. The dotted lines indicate the grouping of harmonics.

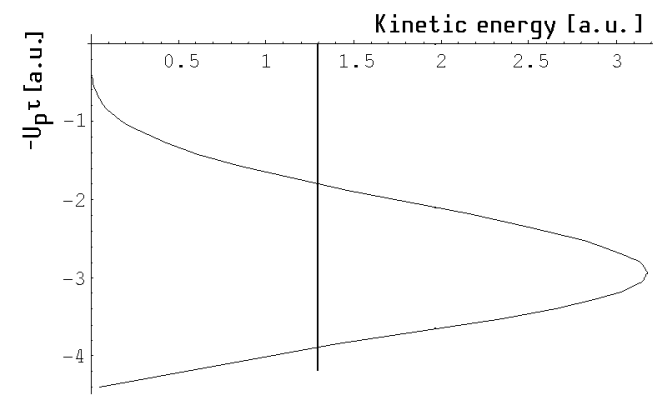

Fig. 5. Classical action $-U_{\mathrm{p}} \tau$ versus kinetic energy at return.

The classical calculation though provides two phases for each kinetic energy, corresponding to a single harmonic order, while in the quantum calculation only one phase per harmonic order survives quantum interference. The upper and lower branches of the curve in Fig. 4 correspond to the long and short electron trajectory, respectively. However, if statistically both branches are present in the quantum calculation (Fig. 3) four narrow peaks per optical cycle will appear in the time domain as indeed found by Antoine et al. Note that on either branch, near the inflexion point, the phase is a quasi linear function of the kinetic energy, i.e. of the harmonic order, and hence the corresponding spectral components are phase-locked.

Now, according to Antoine et al. numerical simulations [5], summation of the fields radiated by all the dipoles contained in the interaction volume, including phase-matching effects, selects one or the other of the two branches depending on the jet position with respect to the laser focus. The precise cause of this amazing effect is difficult to trace back as it results from the role of not only the atomic phase, but also of the geometric phase in a Gaussian beam (so-called Gouy phase) and the plasma and atomic dispersion terms, all of which except the last are intensity-dependent (and therefore space and time dependent). As a consequence of 
this exciting prediction, since apparently one branch of the curve is eliminated and only two narrow temporal peak per optical cycle survive, the maximum contrast is achieved for the resulting subfemtosecond pulse train.

In summary, harmonics are in phase because the classical action along either the long or the short trajectories is a quasi-linear function of intensity (this is primarily a consequence of the non-perturbative aspect of high order harmonic generation) and because of the interplay of the resulting intensity dependent-phase with the other intensity-dependent phases in the macroscopic emission volume.

\section{Determination of the harmonic relative phases}

Since the time domain is not accessible to measurement with enough resolution, one alternative is to seek the information in the Fourier space, i.e. the frequency or energy domain. Véniard et al. [7] have proposed that this phase could be retrieved from a measurement of a three-color, two-photon ionization process. In such a transition, the transition amplitude is proportional to the product of two of the three fields and is a function of their phases. The principle of the method can be sketched as follows: Consider the superposition of two fields of the same amplitude $E_{0}$ at consecutive harmonic frequencies $q \omega$ and $(q+2) \omega(q$ is an odd integer $): E_{0} \exp \left[\mathrm{i}\left(q \omega t+\varphi_{q}\right)\right]$ and $E_{0} \exp \left(\mathrm{i}\left[(q+2) \omega t+\varphi_{q+2}\right]\right)$ and a fundamental field $E_{1} \exp [i(\omega t+\varphi)]$. Because the transition to the final state of energy $(q+1) \omega$ (atomic units) can occur through either the absorption of one photon of the harmonic $q$ followed by absorption of one fundamental photon or one photon of the harmonic $q+2$ and emission of one fundamental photon, the transition amplitude is the sum of two terms

$$
\begin{aligned}
& A^{\prime} E_{0} \exp \left[\mathrm{i}\left(q \omega t+\varphi_{q}\right)\right] E_{1} \exp [\mathrm{i}(\omega t+\varphi)] \\
& \quad+B^{\prime} E_{0} \exp \left(\mathrm{i}\left[(q+2) \omega t+\varphi_{q+2}\right]\right) E_{1} \exp [-\mathrm{i}(\omega t+\varphi)],
\end{aligned}
$$

hence the probability is

$$
A^{2}+B^{2}+2 A B \cos \left(2 \varphi+\varphi_{q}-\varphi_{q+2}+\theta_{q}-\theta_{q+2}\right)
$$

where the two transition matrix elements $A^{\prime}$ and $B^{\prime}$ have been written as $A \exp \left(\mathrm{i} \theta_{q}\right.$ ) and $B \exp \left(\mathrm{i} \theta_{q+2}\right)$, respectively with $A$ and $B$ real. The matrix elements are complex because of the divergence which appears in the summation over intermediate states in the second-order perturbation, two-photon transition as the harmonic photons are larger than the ionization potential.

Equation (2) is the key result of [7] and suggests right away the experimental method: if $\varphi$ is taken as the phase of the fundamental relative to that of the harmonic field of (say) order $q$ (the absolute phase origin is arbitrary) it can be written as $\omega \tau$ where $\tau$ is a delay introduced between the two fields, then Eq. (2) predicts that the two-photon peak in the photoelectron energy spectra, will be modulated by the cosine function of period $\pi / \omega$ (i.e. half the fundamental optical 
cycle) with a phase which is a sum of the phase difference between the two harmonic fields and an atomic phase arising from the two-photon matrix element.

\section{Practical implementation}

The setup has been described in details elsewhere [8, 13]. Briefly, the laser beam ( $6 \mathrm{~mJ}, 40 \mathrm{fs}, 1 \mathrm{KHz}$ Ti:sapphire from the LOA, Palaiseau) laser is split into two collinear parts by an annular mask: one outer annular intense beam used to generate harmonics and an inner, weak flat top beam used as the IR beam of the theory above. The two pulses may be delayed with respect to each other by two glass plates of the same thickness (Fig. 6).

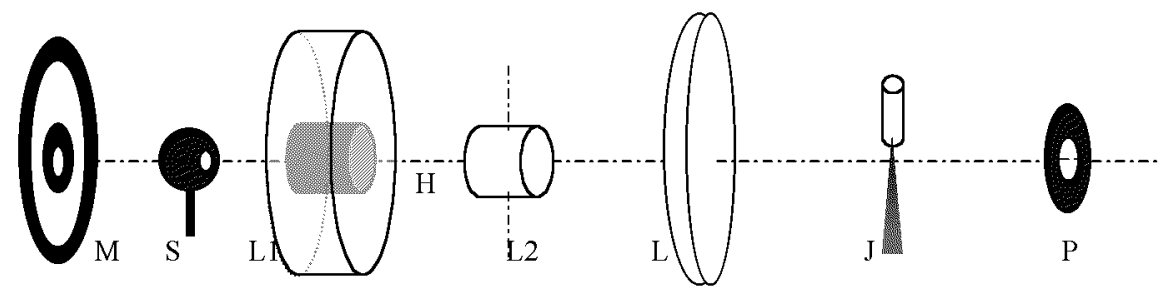

Fig. 6. Co-axial interferometer. The mask $M$ splits the beam into two co-axial beams. Glass plates $L 1$ and $L 2$ control the relative timing of the two pulses while the sphere $S$ controls the energy of the central beam. The central beam travels through the hole $H$ inside $L 1$. Lens $L$ focuses the annular beam into the harmonic jet $J$ but very weakly the central one. Distances between $L$ and $J$ and $J$ and $P$ are not to scale.

Both beams are then focussed by the lens $L$ into a gas jet where harmonics are generated although only the external annular beam actually contributes to the generation since the central beam intensity in the jet is much too low. The harmonic beam and this weak beam, after elimination of the annular beam by a pinhole $P$, are focused into a second gas jet located inside an electron time-of-flight spectrometer where the two-photon ionization takes place. The electron spectra display photoelectron peaks due to harmonics alone and peaks due to two-photon transitions. The first ones are separated by $2 \omega$ (since only odd harmonics are present) and the others (sidebands) are located at mid-distance between them. The same setup may be used for cross-correlation measurements by monitoring the sidebands amplitudes versus temporal delay (for delays of the order of the pulses), for using the ponderomotive streaking of the ionization potential [13] and for the present method which differs from the cross-correlation only in the delay, now of the order of the optical cycle.

A typical result is shown in Fig. 6. The sideband amplitude is indeed (rather strongly) modulated as predicted by Eq. (2) with a period found equal to $1.3 \mathrm{fs}$, i.e. half the fundamental period of $2.67 \mathrm{fs}$. The data can be convincingly fitted by a 
cosine function whose phase knowledge is the first step towards the determination of the sought $\varphi_{q}-\varphi_{q-2}$ difference. Similar results are obtained for the three other sidebands that could be monitored between harmonic 11 and 19. The apparent phases are very close to each other for the first 3 and somewhat different for the last one whose accuracy though suffers from a weaker signal and a poorer statistics. It is interesting to note that no modulation at the fundamental period appears to affect the signal. This is a rather pleasant surprise since such a modulation could be expected for different reasons: (a) the two fundamental fields could interfere in the harmonic jet and, in spite of the large difference in their pulse energies, create a modulation of the harmonic intensity with the fundamental period; (b) some fundamental light from the outer annular beam is scattered from the harmonic jet and passes the pinhole $P$. This light could interfere with the central beam inside the second jet, modulates the IR intensity and hence the two-photon sideband peak. Actually the sideband signal of Fig. 7 is normalized to the total count to smooth out long term variations of the experiment parameters (a typical scan takes about two hours at 60000 laser shots per delay) and therefore modulations at the fundamental period might be eliminated in this process.
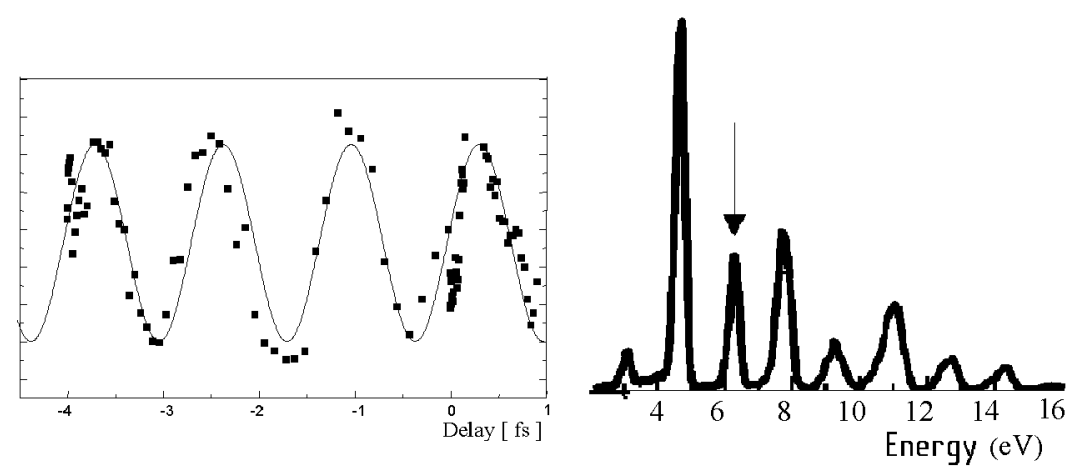

Fig. 7. Typical data showing the sideband (indicated by the arrow on the spectrum on the right) amplitude versus delay. The solid line is a cosine fit.

It is worth stressing that the method measures the phases of the harmonic fields at the second gas jet. Any phase shift introduced by the refocusing mirror adds to that of Eq. (2) and must be subtracted from the measured global phase. This phase can be calculated from the value of the refractive index and/or known optical constants of the reflecting material. A glass spherical mirror covered with a single layer of tungsten has been used. Figure 8 shows the reflectivity and phase shift at normal incidence on a monolayer of tungsten for the range of photon energies corresponding to harmonics 11 to $19(17.75$ to $29.45 \mathrm{eV})$. The phase shift varies by about 1 rad over the whole range.

Another phase must be determined before the phase difference between con- 


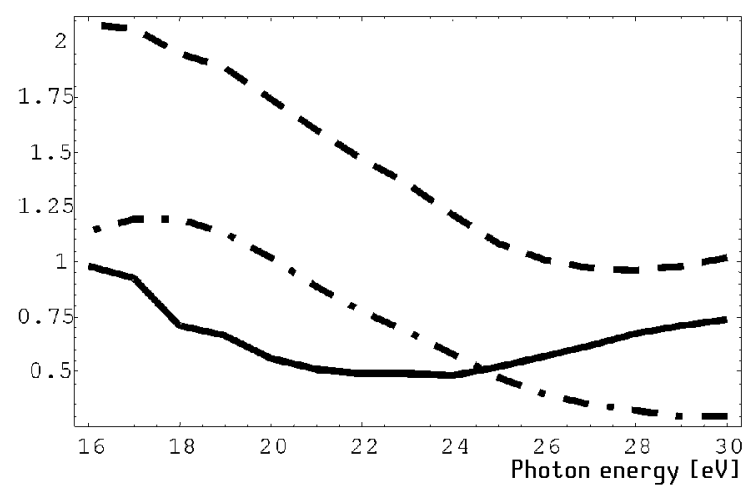

Fig. 8. Real (solid) and imaginary (dot-dash) parts of $W$ refractive index vs. photon energy in $\mathrm{eV}$. Phase shift (rad) is shown by a dashed line. The solid segments indicate the energy of harmonics of the order 11-19.

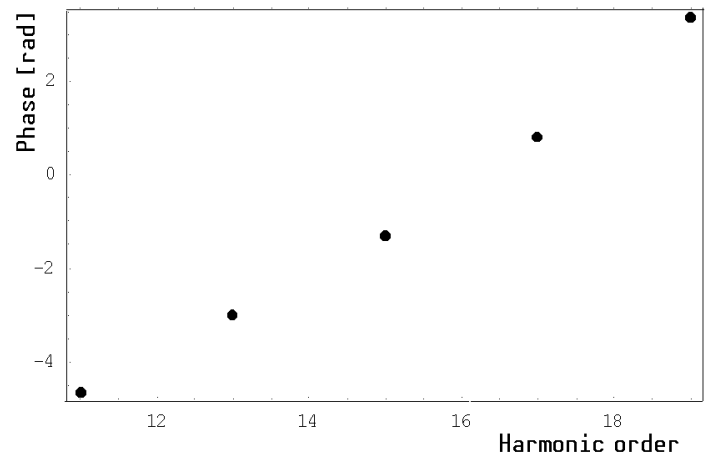

Fig. 9. Harmonic 11-19 phases after correction from the atomic phase and the $W$-mirror phase.

secutive harmonics can be retrieved from the measurement, namely the atomic phase $\theta$ of Eq. (2). This atomic phase, known only from the calculation [9], was calculated within the framework of second-order perturbation theory and then subtracted from the experimental phase with the remarkable result shown in Fig. 9, neglecting the mirror phase shift.

Knowing the spectral phase density and the amplitudes of the harmonics from the associated photoelectron peaks and the documented photoionization cross-sections, the corresponding time profile may be reconstructed [9]. The result is a periodic sequence of eight to ten 250 as pulses (assuming the $10 \mathrm{fs}$ envelope previously determined [14]). In this measurement the highest harmonic order that could be monitored was 19 due to the rapid loss of reflectivity of tungsten at quasi normal incidence. It is anticipated that using a grazing incidence mirror will increase the useful range of the method. Another limit though is the photoionization cross-section which decreases too with the photon energy. However, longer acqui- 

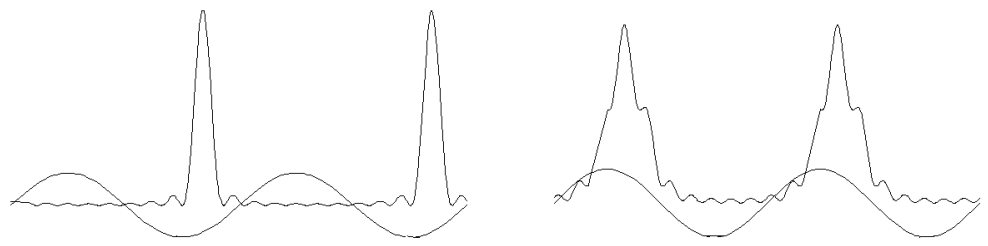

Fig. 10. Simulated pulse trains from harmonics 11-19 of equal amplitudes and a linear spectral phase density (left) and $10 \%$ quadratic term added (right).

sitions should not be a problem at the repetition rate of $1 \mathrm{kHz}$ available with this laser. It may be also anticipated that a larger number of harmonics would then explore some nonlinearity of the curve in Fig. 4. Although the method described in this work is applicable to the extreme UV it is dubious that pulses shorter than 100-150 as will be easily seen with the present setup. Note that a small amount of quadratic distortion of the linear spectral phase density rapidly broadens the pulses as showed by the simulation in Fig. 10 in which a $10 \%$ quadratic term has been added to the linear one.

\section{Single attosecond pulse}

Besides the periodic train of subfemtosecond pulses emitted by a sequence of plateau harmonics of consecutive orders because of the special phase relationship between them, it is naturally desirable to produce a single subfemtosecond pulse. In the case of mode-locked lasers, a similar problem was solved by extracting one pulse from the train with a Pockels cell. The equivalent device would have to open and close in less than $2 \mathrm{fs}$ which is much shorter than available. Rather than extracting one pulse from along train another strategy is to try to produce harmonics with a very short pulse envelope. This can be in principle achieved either by using a very short pump pulse [14] or by limiting the harmonic emission to a small fraction of a longer one.

The shortest $800 \mathrm{~nm}$ pulse achieved as yet is about $5 \mathrm{fs}$. Considering the nonlinearity of harmonic generation, it can be hoped that the harmonic pulses produced with such a pump will be approaching the fs limit. This is indeed what was observed [2] using the technique of the ponderomotive streaking of the ionization potential [13] as a probe: using a 7 fs pump pulse harmonics in the $58-71 \mathrm{eV}$ range produced in neon were found to have a duration of $1.8 \mathrm{fs}$, shorter than the fundamental optical cycle. This approach is limited by the shortest pump pulse that can be efficiently generated and, assuming a 5 fs it is not impossible to envision 1 fs or shorter harmonic pulses that way.

Another proposal is to chirp the polarization form linear to circular and again circular along the pump pulse evolution. This idea is suggested by the extreme sensitivity of harmonic generation efficiency to a small ellipsity in the pump 


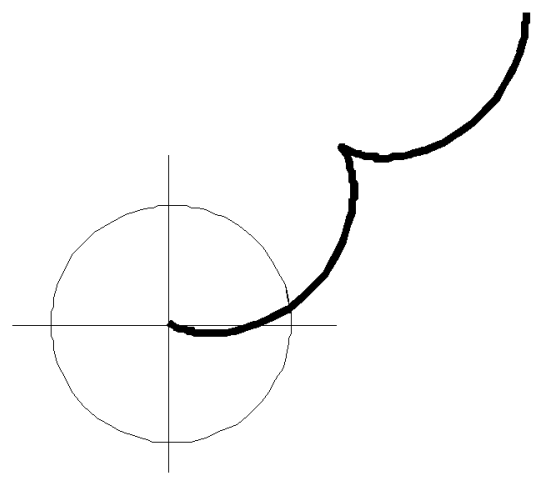

Fig. 11. Electron trajectory (thick line) in the $x y$ plane in a field circularly polarized in the same plane.

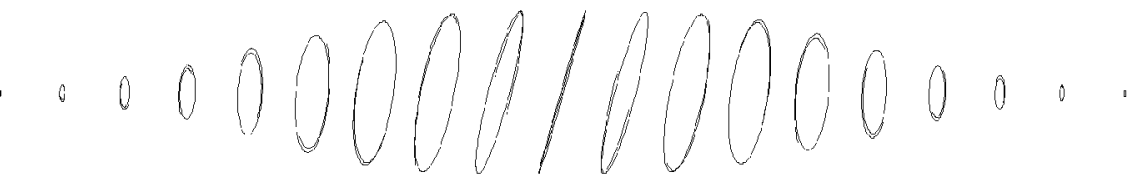

Fig. 12. Polarization chirp produced by superposing two Gaussian pulses linearly polarized along two orthogonal directions and with a temporal delay equal to half their duration. The polarization is linear only for a fraction of the pulse around its maximum.

light (recalling the classical model this stems from the fact that the electron acquires a transverse drift velocity in a circularly polarized field (Fig. 11) and never recombines with its ion). The polarization chirp can in principle be achieved by superposing two linearly polarized fields of slightly different frequencies [15]. Recently a way to achieve the same result has been proposed [16] using just one frequency: superposing two fields circularly polarized with opposite directions with a temporal delay of the order of the pulses duration produce the desired polarization variation (Fig. 12). The rate of the chirp and the intensity during the time the resulting pulse is linearly polarized depend on the delay and the pulse profiles.

\section{Conclusion}

It so turns out that the perhaps naive ideas put forward at the beginning of the nineties [17] and later on confirmed by a more elaborate theory [5] are true and that a linear phase relationship holds between consecutive orders. This prediction was verified in an experiment exploiting the interference between two-photon ionization amplitudes which allowed us to reconstruct the attosecond beating between a group of plateau harmonics generated in argon. The femtosecond barrier is crossed for the first time by such a source delivering, in the case investigated, 
a train of 250 as pulses separated by $1.3 \mathrm{fs}$ (half the fundamental period). It may be expected that pulses twice as short could be easily obtained by using a grazing incidence metallic mirror while 25 as pulses (requiring the beating of 40 consecutive harmonic orders) are not extravagant. Besides, the perspectives for reducing the train to a single pulse through polarization chirping are reasonably good and will be tested shortly by several groups.

\section{References}

[1] T. Brabec, F. Krausz, Rev. Mod. Phys. 52, 545 (2000).

[2] M. Drescher, M. Hentschel, R. Kienberger, G. Tempea, C. Spielman, G. Rieder, P. Corkum, F. Krausz, Science 291, 1923 (2001).

[3] A.V. Sokolov, D.R. Walker, D.D. Yavuz, G.Y. Yin, S.E. Harris, Phys. Rev. Lett. 85, $562(2000)$.

[4] P. Salières, A. L'Huillier, Ph. Antoine, M. Lewenstein, Adv. At. Mol. Opt. Phys. 41,83 (1999).

[5] Ph. Antoine, A. L'Huillier, M. Lewenstein, Phys. Rev. Lett. 77, 1234 (1996).

[6] T. Sekikawa, T. Ohno, T. Yamazaki, Y. Nabekawa, S. Watanabe, Phys. Rev. Lett. 83, 2564 (1999); D. Descamps, L. Roos, C. Delfin, A. L’Huillier, C.-G. Wahlström, Phys. Rev. A 64, 031404(R) (2001).

[7] V. Véniard, R. Taieb, A. Magnet, Phys. Rev. A 54, 721 (1996).

[8] P.M. Paul, E.S. Toma, P. Breger, G. Mullot, F. Augé, Ph. Balcou, H.G. Mullerand, P. Agostini, Science 292, 1689 (2001).

[9] P.B. Corkum, Phys. Rev. Lett. 71, 1994 (1993).

[10] M. Lewenstein, P. Salières, A. L’Huillier, Phys. Rev. A 52, 4747 (1995).

[11] H.G. Muller, Atomic Physics, Eds. H.B. Van Linden van Den Heuvell, J.T.M. Walraven, M.W. Reynolds, Vol. 15, World Scientific, 1997.

[12] P. Salières, B. Carré, L. LeDéroff, F. Grasbon, G.G. Paulus, H. Walther, R. Kopold, W. Becker, D.B. Milosevic, A. Sanpera, M. Lewenstein, Science 292, $902(2001)$.

[13] E. Toma, H.G. Muller, P.M. Paul, P. Breger, C. Leblanc, G. Mullot, G. Chériaux, Phys. Rev. A 62, 061801 (2000).

[14] I.P. Christoff, M.M. Murnane, H.C. Kaptein, Phys. Rev. Lett. 78, 1251 (1996).

[15] P.B. Corkum, N.H. Burnett, M.Y. Ivanov, Opt. Lett. 19, 1870 (1994).

[16] V.T. Platonenko, V.V. Strelkov, QE 28, 749 (1998); E. Constant, Ph.D. Thesis, unpublished (1998).

[17] G. Farkas, C. Toth, Phys. Lett. A 168, 447 (1992); S. Harris, J.J. Macklin, T.W. Hänsch, Opt. Commun. 100, 487 (1993). 